\title{
Triple-Peptide Receptor Targeting In Vitro Allows Detection of All Tested Gut and Bronchial NETs
}

\author{
Jean Claude Reubi and Beatrice Waser \\ Cell Biology and Experimental Cancer Research, Institute of Pathology, University of Berne, Berne, Switzerland
}

\begin{abstract}
A high proportion of gut and bronchial neuroendocrine tumors (NETs) overexpresses somatostatin receptors, especially the $s_{2} t_{2}$ subtype. It has also recently been observed that incretin receptors, namely glucagonlike peptide 1 (GLP-1) and glucose-dependent insulinotropic peptide (GIP) receptors, can be overexpressed in gut and bronchial NETs. However, because not all tumors can express these receptors in sufficient amounts, in vivo imaging with a single radioligand may not always be successful. We therefore evaluated with in vitro methods whether a cocktail of radioligands targeting these 3 receptors would improve tumor labeling. Methods: In vitro receptor autoradiography was performed on $55 \mathrm{NETs}$, comparing in each successive section of tumor the binding with a single radioligand, either ${ }^{125}$-Tyr ${ }^{3}$-octreotide, ${ }^{125}$-GLP-1(7-36)amide, or ${ }^{125}$ I-GIP(1-30), with the binding using a cocktail of all 3 radioligands, given concomitantly under identical experimental conditions. Results: Using the cocktail of radioligands, all tumors without exception showed moderate to very high binding, with a receptor density corresponding to $1,000-10,000 \mathrm{dpm} / \mathrm{mg}$ of tissue; conversely, single-ligand binding, although identifying most tumors as receptor-positive, failed to detect receptors or measured only a low density of receptors below $1,000 \mathrm{dpm} / \mathrm{mg}$ in a significant number of tumors. In addition, the cocktail of radioligands always provided a homogeneous labeling of the whole tumor, whereas single radioligands occasionally showed heterogeneous labeling. Conclusion: The study suggests that the use of a cocktail of 3 radioligands binding to somatostatin receptors, GLP-1 receptors, and GIP receptors would allow detecting virtually all NETs and labeling them homogeneously in vivo, representing a significant improvement for imaging and therapy in NETs.
\end{abstract}

Key Words: multireceptor imaging; neuroendocrine tumors; somatostatin receptors; GLP-1 receptors; incretin receptors

J Nucl Med 2015; 56:613-615

DOI: 10.2967/jnumed.114.153189

$\mathbf{O}$ ver the past 2 decades, in vitro methods have shown that peptide receptors are overexpressed in neuroendocrine tumors (NETs) $(1,2)$. This discovery has had clinical implications for tumor imaging and tumor radiotherapy, in particular for tumors overexpressing the somatostatin receptor subtype $2, \operatorname{sst}_{2}(3,4)$. However, not all NET patients can benefit from the advantages

\footnotetext{
Received Dec. 16, 2014; revision accepted Jan. 21, 2015.

For correspondence or reprints contact: Jean Claude Reubi, Cell Biology and Experimental Cancer Research, Institute of Pathology, University of Berne, P.O. Box 62, Murtenstrasse 31, CH-3010 Berne, Switzerland.

E-mail: reubi@pathology.unibe.ch

Published online Feb. 19, 2015.

COPYRIGHT (c) 2015 by the Society of Nuclear Medicine and Molecular Imaging, Inc.
}

of sst $_{2}$ targeting in diagnosis and therapy, because not all tumors have a high enough $\mathrm{sst}_{2}$ density (2). Therefore, new targets, as alternatives to somatostatin receptors, are being investigated. It has been observed with in vitro techniques that other peptide receptors such as glucagonlike peptide 1 (GLP-1), glucose-dependent insulinotropic peptide (GIP), and cholecystokinin receptors are also overexpressed in NETs (2,5-7). First clinical implications using radioligands derived from these regulatory gut peptides are emerging. For instance, in vivo GLP-1 receptor imaging localizes virtually all benign insulinomas $(8-10)$. But again, unfortunately, not all NETs overexpress these receptors: GLP-1 receptors are indeed more rarely expressed in ileal NETs and malignant insulinomas $(2,9)$. More recently, in vitro receptor autoradiography found that receptors for the GIP are overexpressed in most NETs, including tumors that lack $\mathrm{sst}_{2}($ (6).

It would be a great progress if nuclear medicine could have a diagnostic imaging method that would not miss any NETs and detect more metastases than current methods allow. It would not only permit a more complete picture of the NET distribution in a patient but also be the basis for a more successful targeted radiotherapy.

We hypothesized in the present study that multireceptor targeting may be a possible solution. Because $\mathrm{sst}_{2}$, GLP-1 receptors, and GIP receptors are often overexpressed in NETs, we performed in vitro receptor autography using a cocktail of 3 radioligands, ${ }^{125}{\mathrm{I}-\mathrm{Tyr}^{3} \text {-octreotide, }}^{125} \mathrm{I}$-GLP-1(7-36)amide, and ${ }^{125} \mathrm{I}-$ $\operatorname{GIP}(1-30)$, and compared the results with autoradiography performed with each of the single radioligands in successive sections of a large series of 55 ileal, pancreatic, and bronchial NETs.

The aim of the study was to know whether the use of a cocktail of 3 radioligands would permit not only unequivocal labeling of every individual tumor but also homogeneous labeling of each tumor, in order to overcome the problem of heterogeneous expression of peptide receptors in tumors.

\section{MATERIALS AND METHODS}

\section{Tissues}

Fresh-frozen samples of 55 tumors obtained from surgical resection specimens and tested previously for somatostatin, GLP-1, and GIP receptors $(2,6)$ were used in this study. The 55 tumors consisted of 14 ileal NETs; 29 pancreatic NETs, including 13 insulinomas; and 12 bronchial NETs. The 55 tumors included primaries, metastases, and tumors with low or high differentiation grade. The study conformed to the ethical guidelines of the Institute of Pathology, University of Bern. The institutional review board approved this study, and all subjects gave written informed consent.

\section{Autoradiography}

In vitro receptor autoradiography for $\mathrm{sst}_{2}$, GLP-1, and GIP receptors was performed on successive sections of each tumor for each receptor individually, as described previously $(2,6,7)$. For $s^{2} t_{2}$ 
autoradiography, ${ }^{125} \mathrm{I}_{-\mathrm{Tyr}}{ }^{3}$-octreotide $(15,000 \mathrm{cpm} / 100 \mu \mathrm{L} ; 74,000 \mathrm{GBq}$ $[2,000 \mathrm{Ci}] / \mathrm{mmol}$; Anawa) was used as the radioligand (2). For GLP-1 receptor autoradiography, ${ }^{125} \mathrm{I}$-GLP-1(7-36)amide $(7,500$ cpm/100 $\mu \mathrm{L} ; 74,000 \mathrm{GBq}[2,000 \mathrm{Ci}] / \mathrm{mmol}$; Anawa) was used as the ligand (7). For GIP receptor autoradiography, ${ }^{125} \mathrm{I}-\mathrm{GIP}(1-30)$ $(10,000 \mathrm{cpm} / 100 \mu \mathrm{L} ; 74,000 \mathrm{GBq}[2,000 \mathrm{Ci}] / \mathrm{mmol}$; Anawa) was used (6). For the cocktail autoradiography, performed on sections adjacent to those used for single-receptor autoradiography, all 3 radioligands were given concomitantly, each in the same dose and same amount of radioactivity as for individual single-receptor autoradiography, using the same incubation protocol as above. In the case of homogeneously distributed peptide receptors, the whole sample was quantified; in the case of heterogeneously distributed peptide receptors, only the area with highest density was considered. This study was feasible specifically with these 3 radioligands because the incubation protocols for the autoradiography of somatostatin receptors, GLP-1 receptors, and GIP receptors are identical. Such is not the case for cholecystokinin receptor autoradiography, therefore preventing us from including cholecystokinin radioligands in the present study.

\section{RESULTS}

Figure 1 shows examples of different NETs tested on successive tissue sections with receptor autoradiography using either ${ }^{125} \mathrm{I}_{-} \mathrm{Tyr}^{3}$ octreotide alone, ${ }^{125}$ I-GLP-1(7-36)amide alone, ${ }^{125}$ I-GIP(1-30) alone, or a cocktail of all 3 radioligands. The first example, a benign insulinoma (no. 1), shows that all 3 receptors are expressed in the tumor, although in different densities, and that the cocktail provides a very strong labeling of the whole tumor. The bronchial NET (no. 2) has no GLP-1 receptors, a moderate GIP receptor content, and a high density of somatostatin receptors: again, the cocktail strongly labels the whole tumor. The malignant insulinoma (no. 3) has both somatostatin receptors and GLP-1 receptors but no GIP receptors, and the cocktail provides massive labeling of the whole tumor.

The next 4 cases in Figure 1 show not only that the cocktail autoradiography results in much stronger labeling of the 4 different tumors but also, even more importantly, that the cocktail labeling overcomes the receptor heterogeneity in these tumors. The somatostatin receptors and GIP receptors are heterogeneously expressed in the ileal NET (no. 4), with areas of strong receptor positivity adjacent to receptor-negative areas. The GLP-1 receptors are homogeneously distributed. The cocktail intensively and homogeneously labels the whole tumor. A similar conclusion is obtained for the bronchial NET (no. 5), with the difference being that this tumor lacks GLP-1 receptors. The nonfunctioning pancreatic NET (no. 6) and the bronchial NET (no. 7) are 2 further examples of inhomogeneous receptor distribution (at the single radioligand level) that results, however, in a very strong and homogeneous tumor labeling with the cocktail.

Figure 2 is a quantification of all gut and lung NET receptor data, with each individual tumor being characterized for its respective receptor density, either measured with 1 of the 3 single radioligands or with the cocktail radioligands. The main message of this figure is that, in the cocktail experiment, there are no single tumors lacking receptor binding. All of the tumors show a receptor density between 1,000 and $10,000 \mathrm{dpm} / \mathrm{mg}$ of tissue, which correspond to a moderate to very high receptor density. None of the cases have values around or below $1,000 \mathrm{dpm} / \mathrm{mg}$, corresponding to low density. Conversely, in each of the single-receptor targeting experiments, there are always several cases that completely lack receptors or have a low density only (Fig. 2). The respective receptor profiles as measured by single-receptor binding correspond in general to previously published data $(2,6)$.



FIGURE 1. In vitro receptor autoradiography on successive sections from 7 different NETs: 1 = benign insulinoma; 2 = bronchial NET; 3 = malignant insulinoma; 4 = ileal NET; 5 = bronchial NET; $6=$ nonfunctioning pancreatic NET; 7 = bronchial NET. From left to right are shown hematoxylin- and eosin-stained section (HE), autoradiogram showing ${ }^{125}$-Tyr ${ }^{3}$-octreotide binding, autoradiogram showing ${ }^{125}$ /-GLP-1(7-36)amide binding, autoradiogram showing ${ }^{125} \mathrm{I}-\mathrm{GIP}(1-30)$ binding, and autoradiogram showing cocktail binding of all 3 radioligands. Cocktail labeling is strongly positive in each case, whereas several single-receptor labelings are negative. In all cases, nonspecific binding was negligible and is therefore not shown. $\star=$ areas of receptor heterogeneity with single-ligand labeling that become completely homogeneous with cocktail labeling. Bars $=1 \mathrm{~mm}$.

\section{DISCUSSION}

The in vitro comparison of single-receptor ligand binding using ${ }^{125} \mathrm{I}$-Tyr $^{3}$-octreotide, ${ }^{125} \mathrm{I}$-GLP-1(7-36)amide, or ${ }^{125} \mathrm{I}$-GIP(1-30) versus cocktail binding with all 3 radioligands in NETs shows several important features. First, it is evident, and as such confirms previous reports $(2,6,7)$, that single ligand binding to each of the 3 receptors is good in a large number of NETs. However, there are with each single ligand a significant number of tumors that are not labeled or that have only a low density of receptors, a density that may not be sufficient to allow in vivo detection (11). Conversely, cocktail binding also reveals the expression of receptors, often in high density, for cases in which binding was negative with at least 1 of the single radioligands. On the basis of previous experience on the comparison of somatostatin receptor autoradiography with somatostatin receptor imaging, the high receptor density detected in the tumors of the present study with the cocktail suggests that virtually all these tumors should also become detectable in vivo with the cocktail (11).

Second, the cocktail may overcome the problem of heterogeneous receptor distribution seen with single radioligands, a heterogeneity that is occasionally observed in NETs and that may be a reason for insufficient imaging in vivo and for poorly successful targeted radiotherapy. For the success of targeted radiotherapy, it may indeed be decisive that the radioligand reaches every single cell of a tumor. The general problem of receptor heterogeneity and its consequence for adequate therapy has recently been emphasized (12).

To our knowledge, our study was the first to investigate such a triple receptor in vitro, although the idea for such a possibility had been introduced many years ago $(2,13)$. However, methodologic 


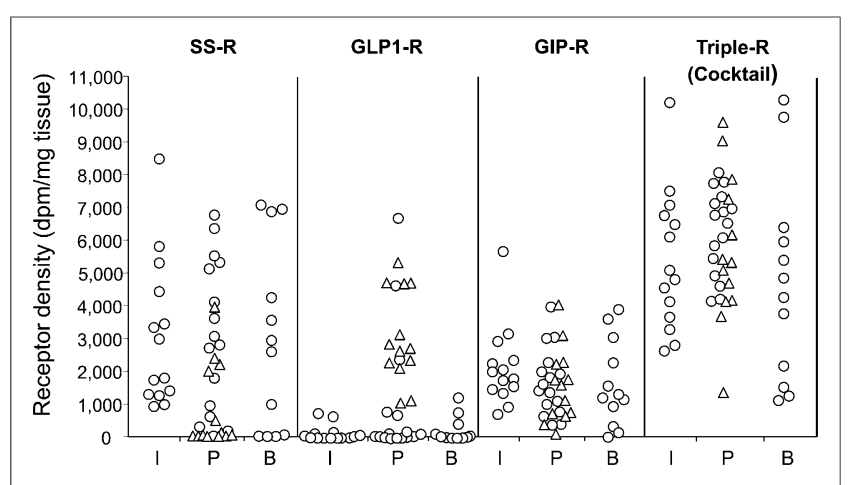

FIGURE 2. Quantification of receptor density (dpm/mg of tissue) in each tumor. Values represent total binding minus nonspecific binding. Mean density of somatostatin receptors for all tumors is $2,542 \pm 319 \mathrm{dpm} / \mathrm{mg}$ of tissue. Mean density of GLP-1-R for all tumors is $1,102 \pm 230 \mathrm{dpm} / \mathrm{mg}$ of tissue. Mean density of GIP-R for all tumors is $1,787 \pm 156 \mathrm{dpm} / \mathrm{mg}$ of tissue. Mean density of cocktail for all tumors is $5,602 \pm 300 \mathrm{dpm} / \mathrm{mg}$ of tissue. $\mathrm{SS}-\mathrm{R}=$ somatostatin receptor autoradiography alone (125/-Tyr ${ }^{3}$-octreotide); GLP-1-R = GLP-1 receptor autoradiography alone (125I-GLP-1(7-36)amide); GIP-R = GIP receptor autoradiography alone (125I-GIP(1-30)); triple $\mathrm{R}=$ receptor autoradiography with all 3 radioligands; $I=$ ileal NET; $P=$ pancreatic $\mathrm{NET}(\triangle=$ insulinomas; $\mathrm{O}=$ all other pancreatic NET); $\mathrm{B}=$ bronchial NET.

considerations made it difficult up to now to have an in vitro cocktail experiment such as the present one performed convincingly. Indeed, to perform a cocktail experiment, it is crucial that the binding protocol be identical for each of the single radioligands, in order to have the cocktail experiment performed under controlled conditions suited to all 3 radioligands at the same time. Although the experimental protocol is different for somatostatin receptors, cholecystokinin receptors, and NPY receptors $(2,13)$, the experimental protocol is, luckily, identical for somatostatin receptors, GLP-1 receptors, and GIP receptors $(2,6,7)$, making it possible to investigate these 3 receptors simultaneously.

What are the consequences of these in vitro data for nuclear medicine? These data indicate that adequate radioligands for the detection of these 3 receptors in vivo have to be chosen: established radioligands exist for somatostatin receptor labeling (chelated octreotide and octreotate derivatives (14), or, more recently, chelated somatostatin receptor antagonist (15)) and for GLP-1 receptor labeling (chelated exendin derivatives (8)); radioligands for GIP receptors are in development, with the first in vivo animal imaging studies giving the proof of concept that GIP receptor targeting is feasible in vivo (10). Better radioligands need to be developed for therapy: specifically, current GLP-1 and GIP analogs have an excessive kidney uptake. Incretin receptor antagonists may be an option; they would also reduce the pharmacologic impact on glucose metabolism when 2 incretin receptor agonists are used concomitantly (8). Also, multivalent ligands may be an option, but it may be difficult to design multivalent ligands with a sufficiently high affinity for each of the 3 receptors.
Then, the optimal radioligand dosage will have to be found and the complex logistic for using 3 different radioligands concomitantly has to be established. Since these ligands are labeled with radiometals, one could envision a procedure in which 3 peptide ligands are labeled in a single reaction vial, which could make multireceptor targeting clinically feasible.

\section{DISCLOSURE}

The costs of publication of this article were defrayed in part by the payment of page charges. Therefore, and solely to indicate this fact, this article is hereby marked "advertisement" in accordance with 18 USC section 1734 . No potential conflict of interest relevant to this article was reported.

\section{REFERENCES}

1. Reubi JC. Peptide receptors as molecular targets for cancer diagnosis and therapy. Endocr Rev. 2003;24:389-427.

2. Reubi JC, Waser B. Concomitant expression of several peptide receptors in neuroendocrine tumors as molecular basis for in vivo multireceptor tumor targeting. Eur J Nucl Med Mol Imaging. 2003;30:781-793.

3. Bodei L, Kidd M, Prasad V, Baum RP, Drozdov I, Modlin IM. The future of nuclear medicine imaging of neuroendocrine tumors: on a clear day one might see forever. Eur J Nucl Med Mol Imaging. 2014;41:2189-2193.

4. Krenning EP, Kwekkeboom DJ, Bakker WH, et al. Somatostatin receptor scintigraphy with [ ${ }^{111} \mathrm{In}$-DTPA-D-Phe $\left.{ }^{1}\right]$ - and $\left[{ }^{123} \mathrm{I}-\mathrm{Tyr}^{3}\right]$-octreotide: the Rotterdam experience with more than 1000 patients. Eur J Nucl Med. 1993;20: 716-731.

5. Reubi JC, Schaer JC, Waser B. Cholecystokinin(CCK)-A and CCK-B/gastrin receptors in human tumors. Cancer Res. 1997;57:1377-1386.

6. Waser B, Rehmann R, Sanchez C, Fourmy D, Reubi JC. Glucose-dependent insulinotropic polypeptide receptors in most gastroenteropancreatic and bronchial neuroendocrine tumors. J Clin Endocrinol Metab. 2012;97:482-488.

7. Körner M, Stockli M, Waser B, Reubi JC. GLP-1 receptor expression in human tumors and human normal tissues: potential for in vivo targeting. $\mathrm{J} \mathrm{Nucl} \mathrm{Med}$. 2007;48:736-743.

8. Christ E, Wild D, Ederer S, et al. Glucagon-like peptide-1 receptor imaging for the localisation of insulinomas: a prospective multicentre imaging study. Lancet Diabetes Endocrinol. 2013;1:115-122.

9. Wild D, Caplin M, Christ E, et al. Glucagon-like peptide-1 vs. somatostatin receptor targeting in malignant insulinomas. J Nucl Med. 2011;52:1073-1078.

10. Wild D, Mäcke H, Christ E, Gloor B, Reubi JC. Glucagon-like peptide 1-receptor scans to localize occult insulinomas. N Engl J Med. 2008;359:766-768.

11. Krenning EP, Kwekkeboom DJ, Pauwels S, Kvols LK, Reubi JC. Somatostatin receptor scintigraphy. In: Freeman LM, ed. Nuclear Medicine Annual 1995. New York, NY: Raven Press; 1995:1-50.

12. Vogelstein B, Papadopoulos N, Velculescu VE, Zhou S, Diaz LA Jr, Kinzler KW. Cancer genome landscapes. Science. 2013;339:1546-1558.

13. Reubi C, Gugger M, Waser B. Coexpressed peptide receptors in breast cancers as molecular basis for in vivo multireceptor tumor targeting. Eur J Nucl Med Mol Imaging. 2002;29:855-862.

14. Wild D, Bomanji JB, Benkert P, et al. Comparison of ${ }^{68} \mathrm{Ga}-\mathrm{DOTANOC}$ and ${ }^{68} \mathrm{Ga}-$ DOTATATE PET/CT within patients with gastroenteropancreatic neuroendocrine tumors. J Nucl Med. 2013;54:364-372.

15. Wild D, Fani M, Fischer R, et al. Comparison of somatostatin receptor agonist and antagonist for peptide receptor radionuclide therapy: a pilot study. $J$ Nucl Med. 2014;55:1248-1252.

16. Gourni E, Waser B, Clerc P, Fourmy D, Reubi JC, Maecke HR. The glucosedependent insulinotropic polypeptide receptor: a novel target for neuroendocrine tumor imaging—first preclinical studies. J Nucl Med. 2014;55:976-982. 